\title{
Microbial Bioremediation of Copper by a Bacillus cereus Strain Isolated from Coastal Waters of Thoothukudi, Tamil Nadu
}

\author{
Rohini R. and Jayalakshmi S.* \\ Centre of Advanced Study in Marine Biology, Annamalai University, India \\ *jayacas@gmail.com
}

\begin{abstract}
Coastal contaminated waters near industrial areas are one of the many environmental compartments where heavy metals and harmful xenobiotics accumulate. Although $\mathrm{Cu}^{2+}$ is an essential micronutrient for most organisms, it exerts several toxic consequences above a threshold concentration. It is known to be a potent toxic element to bacteria and it is one of the most widespread contaminants of the environment. Hence the present study on a copper resistant bacterium isolated from a contaminated coastal water to evaluate its bioremediation potential. The potent $B$. cereus strain was isolated using copper incorporated nutrients agar plate and checked for its antibiotic resistant pattern against commonly used antibiotics. The strain was found to grow up to $600 \mathrm{ppm}$ copper. The level of thiols produced in response to copper was analyzed and the total thiols, non protein and protein thiols were estimated. High total thiol content was observed at 48 hours of incubation with high protein thiols which coincided with high protein content. Optimization parameters for copper accumulation were determined. Regarding optimization, $\mathrm{pH}$ : 6 , temperature: $40^{\circ} \mathrm{C}$, salinity: $10 \%$ and glucose and beef extract were found to be the ideal carbon and nitrogen sources respectively. Resistance of B. cereus against other important heavy metals like mercury, nickel and cadmium, cobalt, chromium and lead were also analyzed. Varied levels of toleration for these metals were also obtained. With all the ideal optimization parameters $49 \%$ of copper accumulation was observed. Thus the strain seemed to have high bioremediation potential against various toxic heavy metal pollutants.
\end{abstract}

Keywords: Bacillus cereus, Bioremediation, heavy metals, Xenobiotics

Proceedings of the International Forestry and Environment Symposium 2016, Department of Forestry and Environmental Science, University of Sri Jayewardenepura, Sri Lanka. 